\title{
EL PROCESO DE DEFINIR EN MATEMÁTICAS. UN CASO: EL TRIÁNGULO
}

\author{
BARROSO CAMPOS, RICARDO \\ Departamento de Didáctica de las Matemáticas. Universidad de Sevilla.
}

\begin{abstract}
SUMMARY
Departing from two protocols that show the difficulties presented to define the triangle in primary school pupils, and through a theoretical framework based on Orton (1990), Herskhovits (1990), Tall and Vinner (1981) and Van Hiele (1981) to establish the meaning that the definition has in Mathematics, we accomplish a summary of definitions of the concept of triangle in various original texts, as well as in material and computer programs.

A short analysis of the definitions presented is accomplished and we offer certain theoretical evaluations to justify the answers given by the students.
\end{abstract}

\section{INTRODUCCIÓN}

\section{Protocolo 1}

- Entrevistador: ¿Qué es un triángulo?

-M. Teresa (Alumna de $4^{\circ}$ de primaria): Hum... (Sin saber qué decir.)

- Ent.: Si te digo que dibujes, en este papel, uno, ¿sabes hacerlo?

-M.T.: ¡Claro que sí!

\section{Protocolo 2}

- Ent.: (Señalando un triángulo rectángulo con los catetos paralelos a los límites del papel, en una lámina con triángulos y figuras mixtas curvilíneas de uno, dos o tres arcos) ¿Es esto un triángulo?

- Estefanía (Alumna de $3^{\circ}$ de primaria): No... (Se queda pensando.)

- Ent.: ¿Por qué...?

- Estefanía: Le falta esta parte (Señalando con el dedo una trayectoria geométrica que completa un isósceles.) para serlo.
Estos dos protocolos realizados con alumnas de un colegio de primaria muestran:

- El primero, las dificultades para expresar una definición personal del concepto (personal concept definition (Tall y Vinner, 1981)) de un polígono fundamental como es el triángulo, aunque se tenga por parte del alumno un determinado componente del esquema conceptual (Azcárate, 1992) (concept image, Tall y Vinner, 1981) y sea capaz de hacer un dibujo.

- El segundo, que la imagen prototípica que la alumna ha fijado en su mente le hace negar que la figura presentada sea un triángulo (porque no se parece a lo que se le ha enseñado a identificar como tal), lo que sitúa a esta alumna en el nivel 1 de Van Hiele, de reconocimiento o visual (Hoffer, 1981; Clements y Battista, 1992). La misma alumna le da atributos irrelevantes como relevantes (ser isósceles, en este caso, como necesario para constituir triángulo) según el esquema de Hershkowitz (1990). 
En este artículo presentamos un análisis de la definición tanto formal como personal de conceptos matemáticos, viendo determinadas implicaciones en el proceso de enseñanza-aprendizaje y centrándonos en el caso del triángulo. Se muestran varias definiciones formales presentadas en textos de diferente procedencia, clase y año de edición, así como la manera en que se puede definir y construir con varios materiales y con tres programas de ordenador.

En el momento actual, los recursos que el profesor de matemáticas de cualquier nivel educativo puede tener en cuenta para planificar sus acciones, llevarlas a cabo en el aula y reflexionar sobre los resultados (fases preactiva, activa y posactiva del proceso enseñanza-aprendizaje) son los textos, materiales y programas de ordenador.

Debido a ello, los consideraremos para mostrar cómo las definiciones formales de triángulo expuestas en los diferentes textos, las utilizaciones de los materiales para construir triángulos y la manera cómo los programas de ordenador (de simulación geométrica) trazan triángulos sobre la pantalla pueden influir tanto en las definiciones personales como en los esquemas conceptuales de los alumnos.

\section{LA DEFINICIÓN DE CONCEPTOS MATE- MÁTICOS}

Según Orton (1990), no se puede esperar que los estudiantes aprendan a través de definiciones, siendo necesario utilizar ejemplos y contraejemplos para la definición de un concepto matemático. Para este autor, se aprende la triangularidad a través de ejemplos de triángulos y del contraste con otras formas.

Probablemente, Estefanía no había recibido suficiente formación en cuanto a ejemplos y contraejemplos del triángulo, llevando las propiedades de determinado tipo (isósceles) a la categoría de condición necesaria para ser triángulo.

Según Tall y Vinner (1981) «La mente humana no es puramente lógica; la forma compleja como funciona varía a menudo de la lógica matemática. El comprender cómo ocurre este proceso, tanto con éxitos como con errores, nos lleva a formular una distinción entre el concepto matemático formalmente conocido y el proceso cognitivo por el que se concibe [...] El esquema conceptual (concept image) describe la estructura cognitiva completa que está asociada al concepto, con inclusión de todas las imágenes mentales, propiedades y procesos asociados al mismo. Se construye a lo largo de los años, mediante experiencias de todo tipo, cambiando cuando el individuo se encuentra con nuevos estímulos y hechos.» (p. 151).

Vinner (1991) señala que «el esquema conceptual es algo no verbal asociado en nuestra mente con el nombre del concepto. Puede ser una representación visual del concepto en el caso de que éste tenga representaciones visuales $[\ldots] \gg$
Tall y Vinner (1981) establecen la definición de un concepto o «concepto definición» (concept definition) como «las palabras usadas para especificarlo. Puede ser personal o formal, siendo esta última la que es aceptada por la comunidad matemática» (p. 152).

M. Teresa carecía del «concepto definición» del triángulo, aunque tenía un componente del esquema conceptual del mismo (una representación mediante un dibujo).

Hershkowitz, 1990, p. 82.

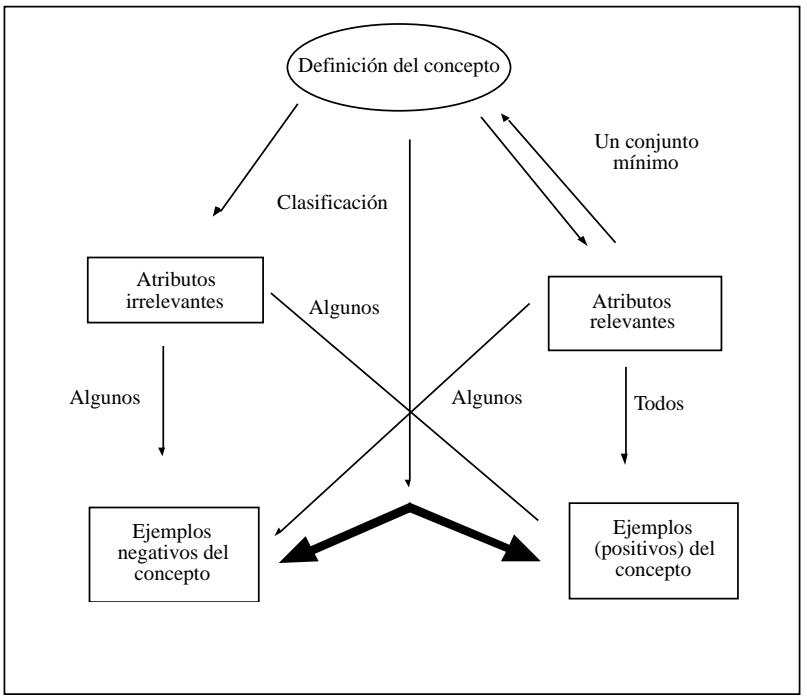

Para Hershkowitz (1990), las relaciones entre los atributos del concepto quedan reflejadas en el esquema anterior. Señala que el prototipo es la base para el juicio prototípico. Para cada concepto, los individuos usan el ejemplo prototípico como un modelo en su juicio de otros casos.

En el protocolo 2 se puede observar claramente que la alumna ha establecido un determinado prototipo (el triángulo isósceles o el equilátero) para sus juicios; es decir, ha atribuido lo que es un atributo irrelevante, el tener dos lados iguales, que poseen algunos triángulos (los isósceles) como relevante y necesario para ser triángulo.

Para determinados análisis se utilizarán los niveles de razonamiento geométrico de van Hiele, el cual consta de cinco niveles (Hoffer, 1981).

Nivel 1: Reconocimiento. El estudiante aprende algo de vocabulario y aprende la forma como un todo.

Nivel 2: Análisis. El estudiante analiza las propiedades de las figuras.

Nivel 3: Ordenación. El estudiante ordena lógicamente las figuras y comprende las interrelaciones y la importancia de definiciones precisas. 
Nivel 4: Deducción. El estudiante comprende el significado de deducción y del papel de los postulados, teoremas y demostraciones.

Nivel 5: Rigor. El estudiante comprende la importancia de precisión en el trato de la fundamentación e interrelaciones entre estructuras.

Estefanía había aprendido vocabulario y consideraba la forma como un todo, lo que la situaba en el nivel 1.

Los cuatro marcos teóricos referenciados, consideramos que están relacionados entre sí. La relación entre los ejemplos y contraejemplos propuestos por Orton y los atributos relevantes e irrelevantes indicados por Herskhovitz, entendemos que pueden dan lugar a un entramado para la construcción del concepto, logrando que se forje en el alumno un esquema conceptual adecuado. También se podría alcanzar con dicho entramado el nivel 2 de van Hiele, de manera que el alumno fuese capaz de establecer y aplicar definiciones mediante el análisis de las propiedades de la figura, en este caso del triángulo.

\section{APROXIMACIÓN MEDIANTE DIVERSOS TEXTOS DE LA DEFINICIÓN DE TRIÁN- GULO}

¿Cómo se define el triángulo en los textos? Revisaremos varios, que tienen diversas aplicaciones y usos, haciendo determinados análisis.

Debido a su carácter excepcional, comenzaremos esta revisión por un libro antológico. El Euclidis Elementorum en la traducción de Federici Commandini en la versión latina de Simson, conservado en la biblioteca universitaria de la universidad hispalense. Según Puertas (1991), ésta señaló un paso a una fase superior del proceso de depuración y rigor de las versiones de los Elementos. Se utilizó sabiamente por Commandini el material que tenía a su disposición: teoninos y escolios que se conservaban en el Vaticano. Fue una versión influyente hasta que apareció la versión de Peyrard (1814-1818).

En su página 4, dice:

Reproduccón de la portada y de la página 4 del Euclidis Elementorum (1756), autorizada por la Biblioteca Universitaria de la Universidad Hispalense.

\section{E U C L I D I S E L E M E N T OR U M

\author{
L I B R I P R I OR E S SEX, \\ IT EM
}

\section{UNDECIMUS ET DUODECIMUS,}

EX VERSIONE IATINA

FE DERICI COMMANDINI;

Sublatis iis quibus olim Libri hi a THE ONE, aliifve, Vitiati funt, Et quiburdam E U C L I D Is Demonftrationibus Reftitutis,

\section{A ROB ER TO S I M SO N, M. D.} In Academiz Glarguenfi Mathefeos Profeffore.

G L A $S G U A E$,

IN AEDIBUS AGADEMICIS

EXCUDEBANT ROBERTUS ET ANDREAS FOULIS ACADEMAE TYPOGRAPHI

M.DCC.LVI.

\section{EUCLIDIS ELEMENTORUM XIX.}

"Segmentum circuli eft figura quaẹ refá linea et circuli circumferen"ria continetur."

$\mathrm{Xx}$.

Rectilineae figurae funt, quae rectis continentur lineis. XXI.

Trilaterae quidem, quae tribus.

XXII.

Qkadrilaterae, quae quatuor.

XXIII.

Multilaterae vero, quae pluribus quam quatuor reetis lineis continentur: XXlv.

Trilaterarum figurarum, acquilatenum eft triangulum, quod tria latera habet acqualia.

XXV.

Ifofceles, quod duo tantum acqualia laters habet.
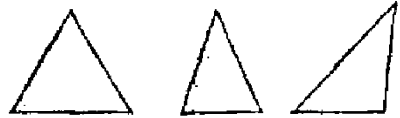

XXVI.

Scalenum vero, quod tria inaequalia habet latera.

XXVII.

Ad haec, trilaterarum figurarum, restangulum quidem trianguhum eft, quod rectim angulam habet.

XXVIII.

Obtufingulam eft, quod obtufum habet anguhum.

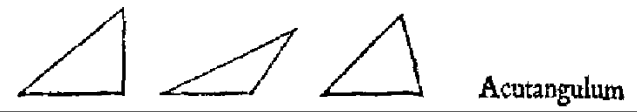


«XX. Rectilinae figurae sunt, quae rectis contenitur lineis.

»XXI. Trilaterae quidem, quae tribus.»

\section{Obras generales sin implicaciones matemáticas}

Comenzamos la revisión desde una perspectiva cultural.

La Enciclopedia Universal Ilustrada Europeo Americana, de Espasa Calpe (1966), dice: «Triángulo, la, F., In., y C. Triangle, It. Triangolo, A. Dreleck, P. y E. Triángulo. (Etim. del latín triangulus), n. Geom. Figura formada por tres líneas que se cortan mutuamente». Esta obra dedica 31 páginas a considerar múltiples propiedades y teoremas. La expresión matemática líneas es imprecisa.

En la Gran Enciclopedia Larousse (1977), se dedica al término casi una página, indicando su etimología, del latín triangulum, y siendo su segunda acepción: «n.m. Figura formada por tres puntos no alineados, y por tres segmentos que los unen dos a dos (los tres puntos son los vértices, y los tres segmentos, los lados)». Esta definición no considera triángulo al «degenerado» (¿Es triángulo la figura formada por tres segmentos sobre tres puntos alineados?...).

En la Encyclopadia Britannica de Benton Publisher (1982), se encuentra: «Triangle, geometrical figure formed by three points that are not on a line.» (Triángulo, figura geométrica formada por tres puntos que no están alineados). Se hace inmediatamente distinción entre el triángulo plano y el esférico. Es la única obra consultada que hace esta distinción en la definición. Al igual que la anterior obra, excluye el triángulo «degenerado».

El Diccionario de la Lengua Española Cumbre de Everest (1990), indica: «Triángulo: m. Figura formada por tres líneas que se cortan.» Esta obra es de uso escolar.

En el Diccionario de la Lengua Española, de la Real Academia Española (1992), el triángulo tiene tres acepciones. La segunda es la que nos interesa «2. m. Geom. Figura formada por tres rectas que se cortan mutuamente formando tres ángulos».

Esta definición es casi la única que incluye el hecho de formar tres ángulos, el cual subyace en la etimología de la palabra (tri-ángulo). Es significativo que al polígono de cuatro lados en lugar de denominarlo cuadrángulo le denominemos cuadrilátero quizá por considerarse que cuadrángulo es una cacofonía.

\section{Obras que son específicas para consulta de matemá- ticas sin ser libros de texto}

En este apartado, revisamos cinco diccionarios o formularios de matemáticas, que pueden formar parte de la biblioteca matemática de un profesor.
En el Formulario Matemático del Bachiller, de SM (1949), en Manuale Sintetico di Matematica, de Bèvesi (1989) y en el Diccionario Oxford de Matemáticas, de Clapham (1992) no aparece ningún epígrafe dedicado expresamente al concepto, aunque sí a algunas de sus propiedades; así, por ejemplo, «expresiones de las medidas de lados y otros elementos en los triángulos [...]» en el primero de ellos.

Interpretamos que la falta de una definición se debe a que, por ser una figura muy elemental, los autores no la han considerado necesaria, dando por supuesto que el lector a quien se dirige el libro conoce el concepto y su definición, comenzando directamente por sus propiedades.

En el Diccionario Básico de Matemáticas, de Díaz (1980), se define como «polígono de tres lados o ángulos».

En Geometría sin esfuerzo, de Sánchez (1983), se tiene como definición y características de un triángulo: «Se entiende por triángulo una porción de plano limitada por tres rectas que se cortan dos a dos. Se representa por el símbolo $\Delta . »$

En esta definición se hace una consideración acerca del símbolo que puede hacer comprender la respuesta dada en el protocolo 2 por la alumna de $3^{\circ}$ de primaria, al visualizar de manera prototípica el triángulo, identificándolo con la imagen que se le muestra.

\section{Obras de texto}

En este apartado consideramos imprescindible hacer una división temporal, debido a que en los años sesenta se produjo un movimiento estructuralista formal en lo que se llamó matemática moderna y que se reflejó, como veremos, en los libros de texto. En primer lugar, revisaremos textos hasta 1960. En segundo lugar, se agruparán textos que se publicaron desde 1961 hasta 1973 y, en tercer lugar, desde 1974 hasta nuestros días.

Desde una perspectiva didáctica, las definiciones que presentan estos textos son un referente de suma importancia para este artículo. A veces, en el desarrollo de la labor del profesor y en el estudio realizado por los alumnos, el libro de texto ha sido el único guía en el proceso de enseñanza-aprendizaje.

\section{a) Obras de texto en el período 1884 a 1960}

En el Tratado de Geometría Elemental, de Cortázar (1884), en cuya página 2 hay una indicación acerca de las «copias» que en aquellos tiempos quizá se prodigasen, se indica: «Habiéndose impreso subrepticiamente en París las obras de Juan Cortázar, se hace presente que todos los ejemplares de dicha procedencia están plagados de errores tan perjudiciales para los que se propongan estudiar en ellos como lo ha sido la falsificación para los intereses del legítimo propietario, que hace la publicación solamente en Madrid.» En ella se define: «Se llama triángulo al polígono que tiene tres lados.» 
En Nociones y Ejercicios de Aritmética y Geometría, de Ruiz (1926), Tratado de Geometría de Tercer Grado, de Bruño (1950) y Matemáticas del Primer Curso de Bachillerato, de Baratech-Estevan (1958), se indica que «triángulo es el polígono de tres lados».

En Matemáticas. Reválida Elemental, $4^{\circ}$, de Marcos y Martínez (1960), obra en cuya portada se observa a dos alumnos que están haciendo un cono con una cartulina, se tiene en la lección 14. «Triángulos 157. Definiciones y clasificación. "Triángulo es una porción de superficie plana limitada por tres rectas que se cortan dos a dos." » Junto a la obra Geometría sin esfuerzo, de Sánchez (1983) son las únicas consultadas en las que aparece en la definición la palabra porción.

En Geometría. Curso Superior, de Bruño (1960), se señala: «Capítulo II: Triángulos. § 1- Generalidades. 57. Definición. "Triángulo es la figura plana formada por una poligonal cerrada de tres lados. O de otro modo, un polígono convexo de tres lados y tres ángulos. O también, la figura formada por tres rectas que se cortan dos a dos."»

Éste es el único texto de esta selección en el que el autor hace referencia de tres definiciones. Es de reseñar el énfasis colocado en la segunda definición al indicar que el polígono debe ser convexo, por dos motivos.

En primer lugar, es la única vez que aparece el concepto polígono convexo en esta revisión de definiciones.

En segundo lugar, habiendo dado el autor previamente la definición de polígono convexo, «Un polígono se llama convexo cuando todos sus puntos están en el mismo semiplano respecto a uno cualquiera de sus lados», es una redundancia incluir dicha característica en la definición.

\section{b) Obras de texto desde 1961 hasta 1973}

En El mundo de los números. $5^{\circ}$ curso, de Osuna y otros (1967), se indica: «Los triángulos y sus propiedades: "El trozo de plano limitado por 3 segmentos de recta se llama triángulo."»

En Fundamentos de geometría, de Coexeter (1971) se dedica el primer capítulo al triángulo, pero sin dar una definición del mismo. Es un estudio espléndido de propiedades y teoremas.

En Matemáticas $5^{\circ}$ curso de educación general básica, de Prada y Cela (1971), se dice en al apartado del triángulo. «1 triángulo. "1.1 Dibuja un ángulo MBP y la recta $b$, que no pase por el vértice $\mathrm{B}$ y que corte a los lados del ángulo como indica la figura 11.2 Colorea en naranja la intersección del ángulo MBP con el semiplano de borde b, que contiene al punto B. Esta intersección se llama triángulo.”»

Había hecho su irrupción la denominada matemática moderna. Los autores indican en la introducción: «Este libro está concebido como un instrumento al servicio del trabajo personal del alumno orientado por el maestro que asume así la noble función de guía y orientador.»

Aunque no está en el objetivo de esta revisión de la definición del triángulo, consideramos significativo de lo que ocurría en la época incluir la definición de polígono realizada por estos autores: «Al conjunto de triángulos, cada uno consecutivo con el siguiente, como lo son los de la figura 1, le llamarás polígono.» (Sin comentarios...)

Figura 1

Prada y Cela, 1971, p. 173.

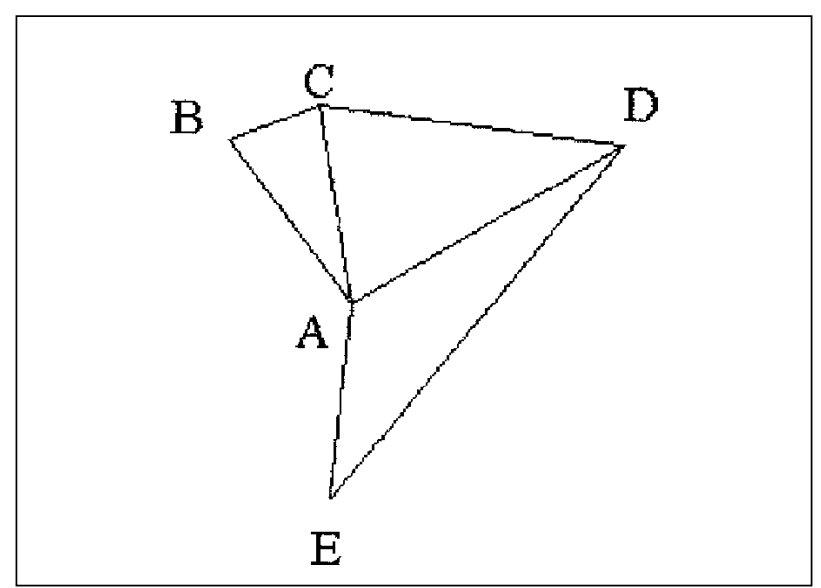

En Matemática moderna, primer curso de segunda enseñanza, de Vázquez y Ramos (1972), se indica sobre triángulos: «Definamos dos puntos sobre una recta y tomemos un tercer punto que no esté contenido en ella y, uniendo los otros puntos con él, veremos que hemos podido definir tres segmentos. Los tres puntos no están alineados. Entonces, podemos decir: si en un plano se tienen tres puntos no alineados y éstos se unen por medio de segmentos, la figura formada se llama triángulo. La unión del conjunto de puntos interiores y los del contorno representan una región triangular.»

Estos autores son, con Jiménez y González (1973), los únicos de todos los textos consultados que diferencian entre contorno (frontera en el caso que veremos a continuación de Jiménez y González) del triángulo y la región interior del mismo.

Además, aparece el concepto matemático conjunto, que de alguna manera aglutinó y marcó la denominada (como el libro en cuestión) matemática moderna. Explícitamente descarta, de los triángulos, el triángulo «degenerado».

En Matemáticas $6^{\circ}$, Libro de Consulta del Alumno, de Jiménez y González (1973), de considera: Concepto de triángulo: «Un ángulo convexo es el conjunto de semirrectas contenidas dentro de la región angular AOB y 


\section{INVESTIGACIÓN DIDÁCTICA}

que tengan como origen el punto de intersección de AO y $\mathrm{BO}$, al cual se le llama vértice. Tracemos un ángulo convexo y una recta que los corte. Observa. Hemos obtenido una figura formada por tres rectas que se cortan dos a dos. Vamos a rayar dicha figura y, la línea que sirve de frontera, de rojo. Los puntos interiores a la línea roja forman una figura que llamaremos triángulo.

Es de reseñar lo «enrevesado» y «exclusivo» de la definición ofrecida.

Para terminar este apartado dedicado al período 196173 , indiquemos que en el prólogo de Matemática 1 , de Rojo y otros (1973) se escribe lo siguiente:

«Hemos realizado este primer texto procurando cumplir, entre otros, los siguientes objetivos: 1. Desarrollar el programa para el primer año del ciclo básico, instrumentando los llamados "temas nuevos de matemática moderna" como enfoque integrador del mismo, más que como tópicos o puntos a exponer por separado [...]»

Indican estos autores: «2.15. Concepto de triángulo. Sean A, B y C tres puntos no alineados de un plano o. La intersección de los semiplanos $\mathrm{S}[\mathrm{AB}, \mathrm{C}], \mathrm{S}[\mathrm{BC}, \mathrm{A}], \mathrm{y}$ $\mathrm{S}[\mathrm{AC}, \mathrm{B}]$ se llama triángulo $\mathrm{ABC}$.»

Es una definición que se distingue por su formalismo y el utilizar el concepto de semiplano que en esta revisión ya ha aparecido dos veces: en Bruño (1960) al definir el polígono convexo y en Prada y Cela (1971).

La definición dada por Rojo y otros (1973) se aparta claramente del resto de las definiciones que están en este artículo. Podría ser incluida en el nivel 4 (de deducción formal) de van Hiele (Hoffer, 1981; Clements y Battista 1992). Explícitamente los autores también excluyen el triángulo «degenerado».

\section{c) Obras de texto desde 1974}

En Matemáticas 1 EGB Ciclo Inicial, de Ferrero y otros (1981), se dice: «Triángulo: Polígono de 3 lados.»

En Matemáticas $3^{\circ}$ EGB Ciclo Medio, de Ramos y otros (1988), se dice: «Clasificación de los polígonos por sus lados: Triángulo: El triángulo tiene 3 lados, 3 vértices y 3 ángulos.» Aparece en esta definición el concepto de ángulo, que no es frecuente.

En Trío, Educación Primaria (Primer Ciclo) de Batalla y otros (1992), se expone: «el triángulo tiene 3 lados y 3 vértices», ante una figura que es un triángulo escaleno con los tres lados inclinados respecto a los límites de la hoja.

Estos autores muestran una serie de dibujos, entre los cuales hay una percha y una señal de tráfico (triángulos),

Educación primaria. Primer ciclo. Batalla y otros (1992). Reproducido con autorización de la editorial Vives.

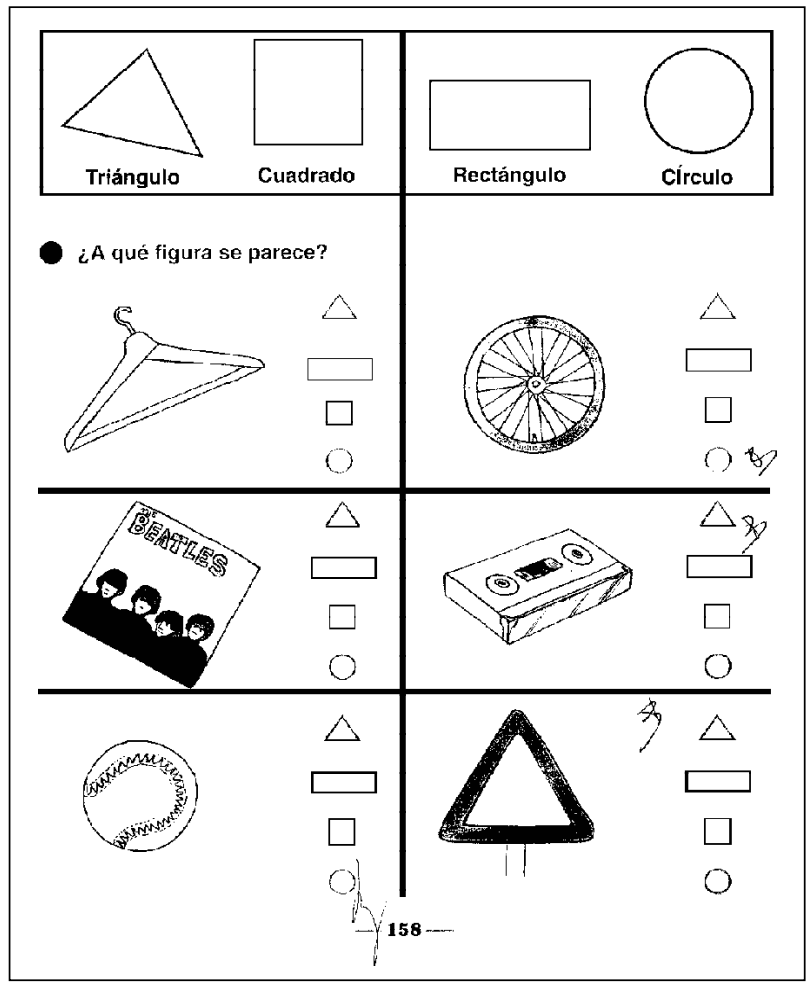

Lados y vértices:

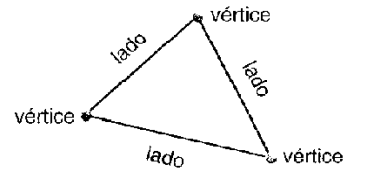

El triángulo tiene:
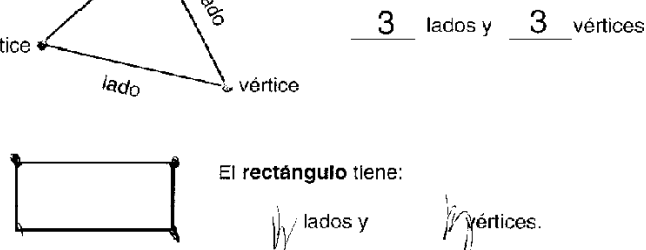

El rectángulo :lene:
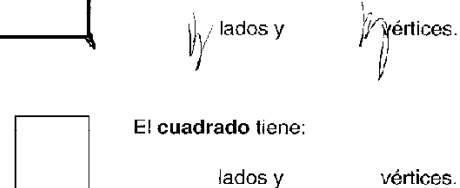

El cuadrado tiene:
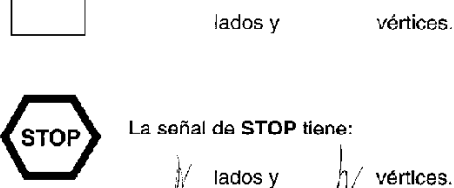

La señal de STOP tiene:

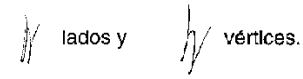

Une los puntos:

$1 \cdot$

- 2

- 3

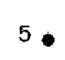

- 4

¿Cuántos lados liene?

- ¿Cuántos vertices tiene?

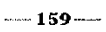


una cinta de casete (rectángulo), una funda de un disco (cuadrado), una pelota de tenis y una rueda de bicicleta (círculos), para que los alumnos contesten a la pregunta: «¿A qué figura se parece?»

Consideramos, dado que este texto se corresponde a $1^{\circ}$ de educación primaria, que la visualización (que, según Hershkowitz [1990], es «la habilidad de representar, transformar, generar, comunicar y reflexionar sobre la información visual») de la figura es necesaria antes de ofrecer una definición.

El tener ante sí ejemplos y contraejemplos, de acuerdo con Orton (1990), permite al alumno captar la esencia del concepto.

También es de reseñar en este texto el uso de atributos irrelevantes como tal, siguiendo el esquema de Hershkowitz (1990). El presentar en la misma hoja tres triángulos, uno equilátero (la señal de ceda el paso), otro isósceles con la base (el lado mayor) inclinada respecto al borde de la hoja (la percha), y otro escaleno, dos círculos, etc., permite al alumno distinguir el triángulo (cualquiera en cualquier posición) de otras figuras geométricas.

En Discovering Geometry. An Inductive Aproach, de Serra (1993), se comienza definiendo el polígono: «A polygon is a closed geometric figure in a plan formed by connecting line segments endpoint to endpoint with each segment interescting exactly two others.» («Un polígono es una figura geométrica cerrada en un plano formada por segmentos conectados por sus extremos y cada uno unido exactamente a otros dos.»)

A continuación, en una tabla, se indica: «You classify polygons by the number of sides they have.» («Clasifique los polígonos por el número de lados que tienen.»)

En el prefacio de este libro, escrito por Shaughnessy, se señala: «Michel Serra ha escrito un libro de geometría genuinamente emocionante. Este libro es único y en él los estudiantes "crean" geometría realmente por sí mismos [...] Los conceptos son en primer lugar introducidos visualmente, después analíticamente, luego inductivamente y, al fin, deductivamente. El espíritu del texto es consistente con las investigaciones recientes sobre el desarrollo del pensamiento geométrico en los jóvenes, particularmente los niveles de pensamiento de van Hiele [...] El autor hace excelente uso de aproximaciones de "contraejemplos y ejemplos" que alientan a los estudiantes a construir sus propias definiciones.»

Terminamos esta revisión con el Cuaderno de Actividades 1, de Fraile y otros (1996), en el que se puede ver en una lámina: «Busca los triángulos, varias copas de árboles, un frontal de una casa, varios tejados triangulares, una señal de ceda el paso, entre otras figuras geométricas, como rectángulos, óvalos y elipses (aproximadamente).»

A esta lámina, que está dirigida a una actividad de alumnos de $1^{\circ}$ de primaria y que, en principio, está bien estructurada, por ser visual, se le pueden hacer dos objeciones desde la perspectiva de los prototipos:

- En primer lugar, todos los triángulos presentados son o equiláteros, o isósceles, por lo que la respuesta dada en el protocolo 2 por Estefanía es coherente con lo que puede haber entendido respecto al triángulo,

- En segundo lugar, todos los triángulos de la lámina tienen la base paralela a la base del papel, con lo que se puede generar el conocimiento de que un atributo que es irrelevante según el esquema de Hershkowitz (1990) anteriormente indicado, pase a ser relevante.

\section{Resumen de las definiciones revisadas en los textos}

\section{a) Definición de triángulo}

Salvo el período $1960-74$, se puede decir que la definición formal dada por los textos revisados de los años 1884-1996 es (lógicamente) similar, siendo un polígono de tres lados.

Es reseñable cómo la denominada matemática moderna llegó a afectar a la definición de un concepto matemático elemental como el que nos ocupa.

Consideramos que el ejemplo de Batalla y otros (1992) es una magnífica muestra de definición de un concepto matemático en el nivel correspondiente y que puede llevar a mejorar el proceso de enseñanza-aprendizaje de las matemáticas.

\section{b) El triángulo como polígono}

En las obras citadas en los apartados anteriores sólo aparece el concepto de polígono en una de las siete definiciones. Quizá el ser obras de consulta general haya influido en los autores de dichos textos a no incluir este término matemático en la definición para que los lectores no versados en matemáticas, que posiblemente sean mayoritarios, no tengan que recurrir a buscarlo. Puede apoyar esta hipótesis el hecho de que no aparezca en las obras del apartado sobre obras generales.

En los libros de texto hasta 1960, de seis definiciones, aparece el concepto de polígono en cinco; sólo Marcos y Martínez (1960) no lo contiene. Sin embargo, este texto, cuando define el polígono, señala: «He aquí los nombres de varios polígonos, presentando una tabla según el número de lados y nombre: 3 lados, triángulo.»

En los cinco libros de texto realizados en la denominada matemática moderna, entre 1961 y 1973, el concepto de polígono está ausente en casi todos, pero creemos que ahora el motivo es bien distinto del comentado sobre las obras de consulta. Es de resaltar en este período la definición hecha de polígono por Prada y Cela (1971) anteriormente citada, al considerarlo como «conjunto de triángulos consecutivos». Quizá el formalismo imperante en la época hizo «borrar» de la definición el hecho de ser un polígono. 
En los cinco libros de texto consultados desde 1974 hasta nuestros días, el concepto de polígono vuelve a estar presente en tres de ellos y, en aquéllos en los que no aparece, está, a nuestro juicio, justificado. Las dos obras en cuestión son Trío, de Batalla y otros (1992) y Cuadernos de Actividades, de Fraile y otros (1996), que están dirigidas a alumnos de primero de primaria, y en este curso debe primar la visualización en el proceso de enseñanza-aprendizaje de la geometría, con lo que $o b$ viar, «decir», que un triángulo es un polígono a tales alumnos es necesario desde un punto de vista didáctico.

\section{c) Atributos de los triángulos}

En casi todas las figuras presentadas, los triángulos tienen un lado horizontal. Ello lleva a los alumnos a considerar como atributo relevante lo que es un atributo irrelevante. Una de las pocas excepciones se encuentra en Batalla y otros (1992), que se ha reproducido.

También es frecuente presentar triángulos isósceles o bien en algunos casos equiláteros, como ocurre en Fraile y otros (1996), con lo que en estos casos el prototipo puede llegar a ser considerado como exclusivo para los juicios.

\section{d) ¿Pueden estar los vértices situados en línea recta?}

La gran mayoría no descarta explícitamente que los vértices puedan estar situados en línea recta, por lo que entiendo que se puede considerar el triángulo «degenerado», con sus vértices en línea recta.

\section{TRIÁNGULO Y MATERIALES}

A continuación se realiza un breve análisis de la utilización de materiales para presentar y construir el triángulo. $\mathrm{Su}$ utilización en clase, creo que puede permitir al alumno avanzar del nivel de reconocimiento -en el que el estudiante aprende algo de vocabulario y aprende la forma como un «todo»- al nivel 2 de análisis -en que el estudiante analiza las propiedades de las figuras (Hoffer, 1981; van Hiele, 1999).

El geoplano, según Dickson y otros (1991), facilita la comprensión de las propiedades de las figuras. Billstein y otros (1990) consideran que el geoplano es un recurso para las actividades que se realizan al nivel 1 visual de Van Hiele, precediendo con actividades intuitivas a la enseñanza de fórmulas de área. Así mismo, la NCTM (1989) lo recomienda para evaluar las destrezas de expresión y comprensión oral de los alumnos.

Las distintas trazas geométricas que pueden presentar los geoplanos (cuadradas, triangulares...) son muy útiles para «construir» y trabajar distintos tipos de triángulos, ya que a través de ellos se pueden estudiar sus propiedades.

El tangram es otro recurso que, según Canals (1994), es útil para ordenar figuras, componer, descomponer $\mathrm{y}$ transformar una figura en otra. Permite construir muy diversos tipos de triángulos.

El «mecano», con varillas de distinta longitud que se enlazan con pequeñas tuercas, permite «construir» triángulos de diversos tipos.

La simple hoja de papel, mediante los cortes pertinentes permite «construir» un triángulo -que se puede manipular-y, haciendo las dobleces oportunas, por ejemplo, trazar las rectas notables del mismo, con lo que se pueden analizar propiedades.

M. Teresa, a propuesta del entrevistador, se ofreció a representar mediante un dibujo con lápiz y papel el triángulo.

\section{TRIÁNGULO Y ORDENADOR}

¿Cómo definen (construyen sobre la pantalla) los programas de ordenador el triángulo? Veamos tres casos: Win-Logo, Cabri-Géomètre y Cabri II nos servirán para el propósito.

\section{a) Win-Logo (1995) de Longman}

Abelson y diSessa (1980) señalan que «lo más importante a tener en cuenta acerca de la geometría de la tortuga es ser una matemática diseñada para la exploración, no sólo para presentar teoremas y demostraciones».

Estas palabras del primer capítulo parecen premonitorias de las consideraciones actuales acerca del proceso de enseñanza-aprendizaje de la geometría (van Hiele, 1999).

Serra (1993) incorpora este programa a sus «Computer activity», en sus «Regular Polygons and Star Polygons with Logo».

El siguiente procedimiento de Win-Logo nos da como resultado un triángulo:

to triangulo: $a$ : ang: $b$; vamos a crear un procedimiento con tres variables

$f d$ : a; le indicamos a la tortuga que avance :a

$r t$ : ang ; la tortuga gira un ángulo de (:ang) ${ }^{\mathbf{o}}$, con lo que el correspondiente ángulo del triángulo tendrá (180 - : ang) ${ }^{\mathbf{r}}$

$f d$ : b; la tortuga avanza: $\mathrm{b}$

home; la tortuga recorre el camino hasta el punto de partida, con lo que el triángulo queda construido

$h t$; la tortuga desaparece para que en la zona de graphics sólo esté el triángulo

end; finalizamos el correspondiente procedimiento. 
Triángulo construido por Win-Logo en el procedimiento triángulo con las variables 3014050 .
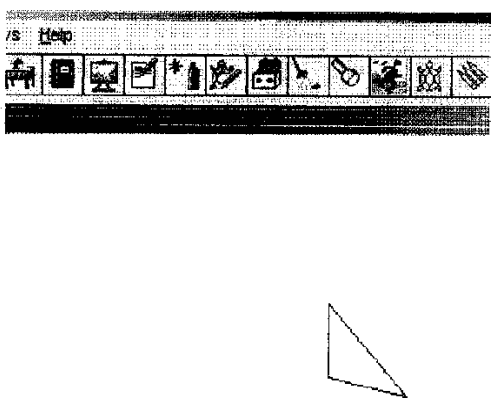

El programa nos indicará en la zona de text: «You have defined triángulo». Así pues, una vez que el procedimiento ha sido preparado, mediante la orden: triángulo 30140 50, por ejemplo, el programa construirá un triángulo.

Las posibilidades que dan las variables introducidas permiten que, por ejemplo, si tenemos un laboratorio de matemáticas, cada alumno construya en su ordenador un triángulo diferente al resto. Es de notar que, si el ángulo de giro es justo de $180^{\circ}$, el triángulo es el «degenerado». Dependiendo de las características de los alumnos, la sesión de trabajo puede continuar de muy distintas formas: ¿Cuánto mide el tercer lado? ¿Cuánto miden los otros dos ángulos?...

\section{b) Cabri-Géomètre (Versión 1.7) (1988-92)}

Este programa de simulación geométrica Cabri (Cahier de Brouillon Interactive (Cuaderno de trabajo interactivo) fue desarrollado en el Laboratorie de Structures Discrètes et de Didactique del Institut d'Informatique et Mathématiques Appliquées de la Université Joseph Fourier de Grenoble, por Jean Marie Laborde y Frank Bellemain. Permite, mediante el menú «Creación», comando «Triángulo», construir un triángulo, bien en puntos creados «sobre la marcha», bien en puntos previamente construidos, ya sea aislados, ya sea sobre un objeto previo. ¿Permite construir un triángulo degenerado? La respuesta es que sí.

Permite también, una vez construido, medir las longitudes de sus lados, marcar sus ángulos y medir sus amplitudes.

Desde una perspectiva que se puede denominar geometría dinámica, permite, «arrastrando» cualquier vértice, modificar el triángulo inicial, con la característica de que todos los elementos, relaciones y propiedades se modifican versus el nuevo triángulo obtenido.

\section{c) Cabri-Géomètre II ${ }^{1}$ (Versión 1.1) (1988-96)}

Para construirlo, podemos ir seleccionando los vértices sobre cualquier figura que tengamos dibujada o bien hacerlo «sobre la marcha».
Usando el cuadro de herramientas «Medir», podemos medir su perímetro y su área, lo que la versión anterior no permitía (medía las longitudes de cada uno de los lados, y el área no tenía presencia en los menús).

En segundo lugar, podemos modificar el triángulo de varias maneras:

- «Arrastrando» los vértices. Si los vértices están aislados, podremos movernos libremente por toda la zona de dibujo (que mediante «Control»y «Clic» del ratón simultáneo podremos ir viéndola). Si están ligados a una determinada figura, ésta restringirá el movimiento del vértice, a no ser que tomemos «Fijar/Liberar» del cuadro de herramientas «Ver», en cuyo caso no podremos «arrastrarlo». Si hemos pedido el perímetro y el área, irán cambiando de valor en función de los triángulos sucesivos.

- «Trasladando, girando, o realizando semejanzas».

Consideramos que estas posibilidades indican que este programa de simulación geométrica toma el triángulo como un «objeto matemático» al poder operar geométricamente con él.

Construcción de un triángulo con Cabri II. Se ha seleccionado un vértice en la zona de dibujo «independiente», otro sobre un objeto (el segmento) y el tercero nos pregunta si se desea que esté sobre otro objeto (la circunferencia).

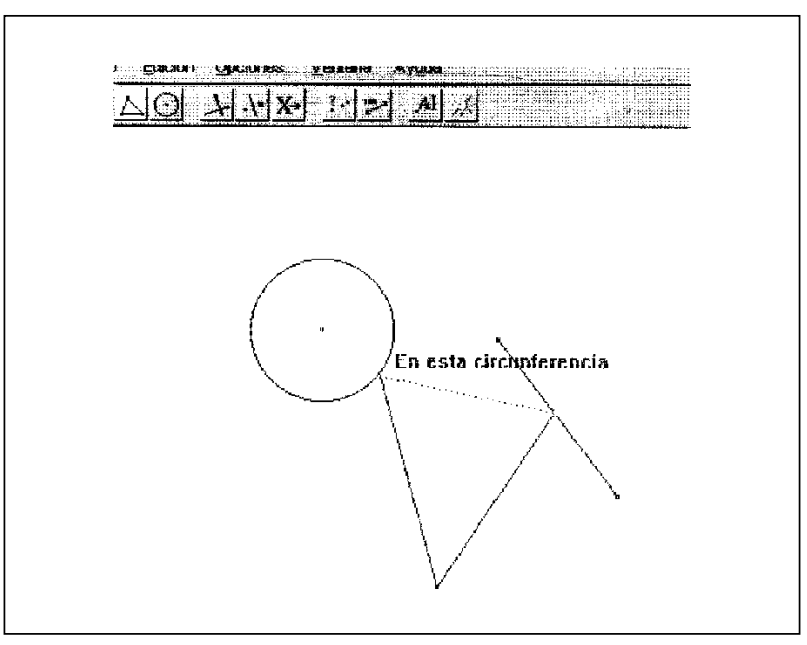

El triángulo es una fuente inagotable de propiedades y teoremas. El teorema de Morley (Coexeter, 1971), que se descubrió a principios del siglo $\mathrm{xx}$, indica que, dado cualquier triángulo, si trazamos las trisectrices de los tres ángulos y buscamos los tres puntos de intersección más cercanos a los lados, estos puntos construyen un triángulo equilátero. El programa Cabri II incorpora por defecto una macro (Trisecta.mac) para la trisección de ángulos y una figura presentando el teorema (Trisec.fig).

Con relación a los protocolos que han sido hilo conductor de este artículo, las alumnas de $3^{\circ}$ y $4^{\circ}$ de primaria no 
habían realizado ninguna sesión de laboratorio informático de matemáticas, lo que es lógico en cuanto a Cabri, en sus dos versiones.

Consideramos que Cabri es para alumnos de $6^{\circ}$ de primaria en adelante, debido a las implicaciones geométricas que tienen sus menús y comandos (bisectriz, mediatriz, lugar geométrico...).

En cuanto a Win-Logo, según Abelson y diSessa (1980) es un lenguaje que puede servir para trabajar a multitud de estudiantes, desde preescolar hasta alumnos de posdoctorado. Así, pues, consideramos que sí podían haber tenido experiencias con él, pero tampoco habían tenido posibilidades de utilizarlo.

\section{CONCLUSIONES}

Hemos realizado un estudio del triángulo desde la perspectiva de la definición ofrecida del concepto en diversos textos, construcción con diversos materiales y por programas de ordenador.

Los textos presentan definiciones en función del lector y del momento en que se escriben, habiendo un paréntesis temporal en la época de la denominada matemática moderna.

Los materiales analizados (tangram, mecano, papel y geoplano) no tienen las mismas características, y cada uno tiene posibilidades didácticas diferentes, siendo el profesor el que, teniendo en cuenta el contexto escolar, delimite aquellas características que desee resaltar para obtener un fin adecuado.

\section{REFERENCIAS BIBLIOGRÁFICAS}

ABELSON, H., y DISESSA, A. (1980). Turtle Geometry. The Computeras a Mediumfor Exploring Mathematics. Cambridge: The MIT Press.

AZCÁRATE, C. (1992). Estudio de los esquemas conceptuales y de los perfiles de unos alumnos de segundo de BUP en relación con el concepto de pendiente de una recta. Epsilon, 24 , pp. 9-22.
En cuanto a los programas informáticos, considero que son un estímulo y que un aula convenientemente adecuada puede convertirse en un laboratorio de matemáticas, donde el alumno experimente, haga conjeturas, explore, se equivoque, aclare sus ideas, verifique sus aciertos, aprenda conceptos, compruebe teoremas.

La correcta integración en el aula de los textos, materiales y programas de ordenador puede permitir unas definiciones matemáticas que superen las dificultades de:

- considerar atributos exclusivos de determinados conceptos específicos (como el tener dos lados iguales) como atributos de un concepto como, por ejemplo, el triángulo,

- tener un esquema conceptual muy limitado y poder tenerlo lo más completo posible.

\section{AGRADECIMIENTOS}

Deseo agradecer al profesor José María Gavilán Izquierdo sus sugerencias durante la redacción del artículo, así como las recomendaciones de los dos asesores de la revista.

\section{NOTA}

${ }^{1}$ Deseo agradecer al ICE de la Universidad de Sevilla la ayuda prestada el curso 1997-98 al Proyecto Software en el aprendizaje de las matemáticas, dirigido por J.M. Gavilán y que contó con la participación de A. Ariza, A. Sánchez, y R. Barroso, para la licencia del programa.
BARATECH-ESTEVAN (1958). Matemáticas del primer curso de bachillerato. Zaragoza: Librería General.

BATALLA, E. et al. (1992). Trío primer trimestre. Educación primaria. Primer ciclo. Barcelona: Vicens Vives.

BÈVESI, M. (1989). Manuale Sintetico di Matematica. Milán: Bignami. 
BILLSTEIN, R., LIBESKIND, S. y LOTT, J.W. (1990). A Problem Solving Approach to Mathematics for Elementary School Teachers. Redwood City: The Benjamin/Cummings Publishing Company, Inc.

BRUÑO, G.M. (1950). Tratado de geometría. Tercer grado. Madrid: Bruño.

BRUÑO, G.M. (1960). Geometría. Curso superior. Madrid: Bruño:

CABRI GÉOMÈTRE. VERSIÓN 1.7 (1988-92). CNRS. Grenoble: Université Joseph Fourier.

CABRI GÉOMÈTRE II. VERSIÓN 1.1 (1988-96). CNRS Grenoble: Université Joseph Fourier.

CANALS, M.A. (1994). Introducción de la medida de superficie en la escuela primaria en Actas de la VI Jornadas de Enseñanza y Aprendizaje de las Matemáticas. Sociedad Extremeña de Educación Matemática «Ventura Reyes Prósper», Federación Española de Sociedades de Profesores de Matemáticas: Badajoz.

CLAPHAM, C. (1992). Diccionario Oxford de matemáticas. Madrid: Celeste Ediciones.

CLEMENTS, D.H. y BATTISTA, M.T. (1992). Geometry and Spatial Reasoning, en Grows, J. (ed.). Handbook of Research on Mathematics Teaching and Learning. Nueva York: Macmillan Publishing Company.

COEXETER,H.S.M.(1971).Fundamentos de geometría. México: Limusa-Wiley, SA.

CORTÁZAR, J.(1884).Tratado de geometría elemental. Madrid: Librería de Hernando.

DÍAZ, M. (1980).Diccionario básico de matemáticas. Madrid: Anaya.

DICCIONARIO DE LA LENGUA ESPAÑOLA, REAL ACADEMIA ESPAÑOLA (1992). Madrid: Espasa Calpe.

DICCIONARIO DE LA LENGUA ESPAÑOLA CUMBRE: (1990). León: Everest.

DICKSON,L.,BROWN, M.y GIBSON, O.(1991). Elaprendizaje de las matemáticas. Barcelona: Labor/MEC.

ENCYCLOPÆDIA BRITANNICA (1982). Helen Hemingway. Chicago: Benton Publisher.

ENCICLOPEDIA UNIVERSAL ILUSTRADA EUROPEO AMERICANA. EDITORIAL (1966). Madrid: Espasa Calpe.

FERRERO, L., GIL, J. y ROLDÁN, G. (1981). Matemáticas 1. EGB Ciclo Inicial. Madrid: Santillana.

FORMULARIO MATEMÁTICO DEL BACHILLER (1949). San Sebastián: Ediciones SM.

FRAILE, J. et al. (1996). Cuaderno de actividades 1 Matemáticas Ir Curso educación primaria, primer ciclo. Barcelona: Vicens Vives.
GRAN ENCICLOPEDIA LAROUSSE (1977). Barcelona: Planeta.

HERSHKOWITZ, R. (1990). Psychological Aspects of Learning Geometry, en Nesher, P. y Kilpatrick, J. (eds.). Mathematics and Cognition. Cambridge: Cambridge University Press.

HOFFER, A. (1981). Geometry is more than Proof. Mathematics Teacher, pp. 11-18

JIMÉNEZ, L. y GONZÁLEZ, A. (1973). Matemáticas $6^{o}$. Libro de consulta del alumno. Salamanca: Anaya.

MARCOS, C. y MARTÍNEZ, J. (1960). Matemáticas. Reválida elemental, $4^{\circ}$. Madrid: SM.

NCTM (1989). Curriculum and Evaluation Standards for School Mathematics: Reston, VA. Traducción cast.: Estándares curriculares y de evaluación para la educación matemática. Sevilla: SAEM Thales.

ORTON, A. (1990). Didáctica de las matemáticas. Madrid: Ediciones Morata/MEC.

OSUNA, D., BERDIÓN, A. y GUARDEÑO, A. (1967). El mundo de los números. $5^{\circ}$ curso. Salamanca: Anaya.

PRADA, D. y CELA, P. (1971). Matemáticas $5^{\circ}$ Curso de Educación General Básica. Madrid: Narcea SA.

PUERTAS, M.L. (1991). Euclides. Elementos. Libros I-IV (Traducción y notas). Madrid: Gredos.

RAMOS, A., GIL, J., GARCÍA, P. y ROLDÁN, G. (1988). Matemáticas 3, EGB Ciclo Medio. Madrid: Santillana.

ROJO, A., SÁNCHEZ, S. y GRECO, M. (1973). Matemática 1. Buenos Aires: El Ateneo.

RUIZ, A. (1926). Nociones y ejercicios de aritmética y geometría. Zaragoza: Artes Gráficas Casañal.

SÁNCHEZ, M. (1983). Geometría sin esfuerzo. Bilbao: Playor.

SERRA, M. (1993). Discovering Geometry. An Inductive Approach. Berkeley: Key Curriculum Press.

TALL, D. y VINNER, S. (1981). Concept Image and Concept Definition in Mathematics with Particular Reference to Limits and Continuity. Educational Studies in Mathematics, 12 , pp 151-169.

VAN HIELE, P.M. (1999). Developing Geometric Thinking through ActivitiesThat Begin with Play. Teaching Children Mathematics, pp. 310-316.

VINNER, S. (1981). The Role of Definitions in Teaching and Learning of Mathematicas, en Tall, D. (ed.). Advanced Mathematical Thinking. Dordrecht: Kluwer Academic Publishers.

VÁZQUEZ, R. y RAMOS, C. (1972). Matemática moderna. Segunda enseñanza. Primer curso. México: Trillas.

WIN-LOGO (1995). Longman Logotron. Cambridge: IDEA, $\mathrm{I}+\mathrm{D}, \mathrm{SA}$.

[Artículo recibido en marzo de 1999 y aceptado en febrero de 2000.] 